\title{
Suporte social de cuidadores de crianças com Síndrome de Down
}

\author{
Luciana Krauss Rezende* \\ Silvana Maria Blascovi de Assis** \\ Luiz Fernando Barca***
}

\section{Resumo}

O objetivo desse estudo foi conhecer a percepção do suporte social de cuidadores de crianças com síndrome de Down (SD), em relação ao grau de satisfação e ao número de pessoas suportivas na região sul do estado de Minas Gerais e estabelecer a relação entre eles. Este é um estudo de análise quantitativa e de caráter clínico, exploratório e descritivo do tipo corte transversal. A população estudada foi composta por 50 pessoas de ambos os gêneros, em sua maioria as mães ( $92 \%)$, cuidadoras de crianças com SD, com idade entre 6 meses a 7 anos e meio, frequentadores regulares de instituições especializadas. Para o grupo estudado, a grande maioria das respostas indicativas de pessoas suportivas apontou a falta de uma pessoa de referência, sendo assinalada a resposta "ninguém". Em seguida, aparecem o marido, filho, pais, irmãos, amigos, esposa e médico da criança. A análise da correlação entre o número de pessoas tidas como suportivas e a satisfação com o suporte social mostrou uma satisfação entre fraca e moderada denotando que esses cuidadores não puderam contar com uma rede de suporte social eficiente no cuidado dos filhos com SD. Considera-se pertinente o incremento de ações e incentivos governamentais e não-governamentais para o desenvolvimento de equipes profissionais e de instituições especializadas, voltadas ao atendimento, à reabilitação e a inclusão de crianças com SD e suas famílias na sociedade. As famílias precisam de programas de apoio apropriados para potencializarem, ao máximo, suas próprias capacidades.

Palavras-chave: Suporte social; Cuidadores; Síndrome de Down.

\footnotetext{
* Professora no curso de Fisioterapia na Fundação de Ensino e Pesquisa de Itajubá. Doutoranda da Universidade Presbiteriana Mackenzie. Itajubá, Minas Gerais, Brasil.

* Professora do Programa de Mestrado e Doutorado em Distúrbios do Desenvolvimento pela Universidade Presbiteriana Mackenzie. São Paulo, São Paulo, Brasil.

*** Professor da Universidade Federal de Itajubá. Itajubá, Minas Gerais, Brasil.
} 


\title{
Social support for caregivers of children with Down's Syndrome
}

\begin{abstract}
The objective of this study was to know the perception of social support for caregivers of children with Down's syndrome (DS) in relation to the degree of satisfaction and the number of people to support in the southern region of the State of Minas Gerais and establish the relationship between them. This is a study of quantitative analysis, exploratory and descriptive character, and clinical type cross-section. The study population consisted of 50 people from both genres, mostly mothers (92), caregivers of children with DS, aged 6 months and 7 and a half years, regular goers of specialized agencies. To the group studied, the vast majority of responses indicating supportive people pointed out the lack of a reference person, being ticked the answer "no". Then appear the husband, son, parents, siblings, friends, wife and children's doctor. The analysis of the correlation between the number of supportive people and satisfaction with social support showed satisfaction among low and moderate denoting that these caregivers could not count on a network of efficient social support in caring for children with DS. It is considered appropriate to increase actions and governmental and non-governmental incentives for the development of professional teams and specialized institutions focused care, rehabilitation and inclusion of children with DS and their families into society. Families need appropriate support programs in order to foster their capabilities.
\end{abstract}

Keywords: Social support; Caregivers; Down's Syndrome.

\section{Introdução}

Conhecida como a alteração genética de maior ocorrência em todo o mundo, a síndrome de Down (SD) é caracterizada por algumas condições que oferecem riscos ao desenvolvimento, como alterações cardíacas, hipotonia, déficit intelectual, entre outras (WISEMAN et al., 2009). Essa condição acaba impondo aos pais e familiares uma série de dificuldades. Segundo levantamento realizado por DESSEN; SILVA (2000), dentre os estudos científicos voltados à deficiência mental, a maioria focaliza a vivência emocional dos familiares, visando seu bem-estar emocional (COUTO; TACHIBANA; AIELLO-VAISBERGl, 2007).

O termo Deficiência Intelectual (DI) surgiu em 2002, proposto pela American Association of Mental Retardation (AAMR), que passou em seguida a ser denominada American Association on Intellectual and Developmental Disabilities (AAIDD); esta denominação passa a considerar a interação dinâmica existentes entre as capacidades funcionais do indivíduo e o meio social no qual está inserido (PAN, 2008). 
A adoção do termo DI implica uma compreensão consistente da deficiência com uma base ecológica e uma perspectiva multidimensional e requer que a sociedade responda com intervenções que foquem nos pontos fortes individuais e que enfatizem o papel dos suportes para melhorar o funcionamento humano. Deve-se reconhecer que a manifestação da DI envolve o engajamento entre a habilidade intelectual, comportamento adaptativo, saúde, participação, contexto e suporte individualizado (WEHMEYER et al., 2008).

Cabe destacar que a Convenção Internacional sobre os Direitos da Pessoa com Deficiência referendada pelo governo brasileiro por meio do decreto 6949/2009 também utiliza o conceito de DI, bem como a Política Nacional de Educação Especial na Perspectiva da Educação Inclusiva.

A Educação Especial deve ser entendida como um conjunto de recursos educacionais que servem de apoio e complemento, garantindo a educação formal àqueles que apresentam necessidades educacionais específicas, diferentes da maioria do alunado que frequenta a rede regular de ensino (MAZZOTTA, 1993). Alunos com SD pertencem ao grupo dos que possuem necessidades especiais, portanto, o atendimento educacional deve envolver a participação da família de modo articulado com as demais políticas públicas (GARCIA; MICHELS, 2011).

A SD é facilmente diagnosticada no período imediato ao nascimento, devido às suas características peculiares, e a notícia transmitida aos pais logo nos primeiros dias de vida da criança. Há alguns anos, vem sendo cada vez mais frequente o conhecimento desse diagnóstico ainda no período gestacional, em decorrência dos exames cada vez mais precisos para a identificação da síndrome, o que traz impacto sobre toda a família (ALDERSON, 2001 \& MOST et al., 2006). O confronto do nascimento entre o bebê imaginado e o real, quando a imagem não corresponde à idealizada, como no caso de crianças com SD (PEREIRA-SILVA \& DESSEN, 2006; BUZATTO \& BERESIN, 2008; CORRICE \& GLIDDEN, 2009), traz respostas de negação ou de aceitação que podem refletir no vínculo que é estabelecido e, consequentemente, nos cuidados dispensados ao filho, bem como no processo de desenvolvimento da criança (CORRICE \& GLIDDEN, 2009; SUNELAITIS et al., 2007).

Em se tratando do nascimento de uma criança com deficiência, o momento é de grande impacto para o casal, causando uma desestruturação e interrupção (em alguns casos de forma traumática) da estabilidade familiar. (DESSEN; SILVA, 2000; CUNHA; et. al, 2010)

A maioria dos estudos conta com as mães como informantes. Um dos argumentos para a utilização do relato das mães como representante do sistema familiar é que o papel de principal cuidador é realizado por ela, o que implica a responsabilidade prática de viabilizar os tratamentos necessários para a criança e dedicar maior tempo aos cuidados. Essa divisão de trabalho tem sido o caminho desde os anos 60 e mudanças não foram encontradas quando as mulheres entraram na força de trabalho. Ressaltam, ainda, que são as mães que fazem acomodações em termos de papéis e 
tempo utilizado destinado a cumprir as responsabilidades associadas com cuidado da criança com necessidades especiais. A atenção à saúde dos cuidadores de crianças com necessidades especiais é uma preocupação recente. Nos anos 80 , os estudos voltaram-se para a investigação do papel dos cuidados dispensados à criança, como também objetivaram a compreensão e identificação de estratégias de coping utilizados pelos cuidadores. (MATSUKURA et al., 2007)

O suporte social pode ser definido como o "conjunto de pessoas significativas para o individuo, que o diferencia da massa anônima da sociedade." Redes de suporte social também podem ser conceituadas como "conjuntos hierarquizados de pessoas que mantêm entre si laços típicos das relações de dar e receber". Essas relações existem ao longo de todo o ciclo vital, porém sua estrutura e funções sofrem alterações (SLUZKI, 1997).

Alguns autores comentam sobre o interesse no estudo do suporte social em função de diversos fatores, dentre eles a importância de achados referentes à relação do suporte social, com indicadores de presença/ausência de diversas doenças e, no caso da presença da doença, a relação do suporte com as previsões de prognóstico e restabelecimento do individuo (MATSUKURA; MARTURANO; OISHI, 2002). Para o mesmo autor, de fato, vários estudos têm apontado a associação entre suporte social e níveis de saúde e/ou a presença de suporte social funcionando como agente protetor, frente ao risco de doenças induzidas por estresse.

Matsukura; Marturano; Oishi (2002), apresentaram uma estrutura teórica dos aspectos presentes no domínio do suporte social que podem estar relacionados à saúde ou ao estresse. Os três grandes aspectos propostos seriam: Relacionamentos sociais (existência, quantidade, tipo); Suporte Social (tipo, fonte, quantidade ou qualidade); Rede Social (tamanho, densidade, reciprocidade, intensidade, dentre outros).

Para o mesmo autor, em relação ao segundo grande aspecto, considera-se "fonte" a identificação do relacionamento estabelecido com o provedor de suporte, como por exemplo, família e amigos. O conceito de redes foi originalmente criado e desenvolvido no âmbito da sociologia antropológica. Sociólogos defendem a noção de que as redes têm algum poder sobre o comportamento social das pessoas envolvidas (SIQUEIRA, 2008).

Ressalta-se que o suporte social deve ser considerado como um metaconstruto com três componentes conceituais distintos, ou seja, recursos de rede de suporte, comportamento suportivo e as avaliações subjetivas de suporte. Sob o enfoque ecológico, os recursos das redes sociais apresentam um "rendimento sustentável", ou seja, podem ser desenvolvidos, mantidos, cuidados e estimulados ou deteriorados, negligenciados e até destruídos, em função de variáveis como características dos indivíduos que compõem a rede, fatores ambientais ou culturais e interações entre essas variáveis (MATSUKURA; MARTURANO; OISHI, 2002). 
Para Siqueira (2008), a importância das redes na construção social reside no pressuposto de que elas preenchem as necessidades individuais, criando para seus integrantes inúmeras oportunidades de manter sua identidade social, receber apoio, ajuda material, serviços, informações e novos contatos sociais.

Para o mesmo autor, na visão psicológica defendida por COBB (1976), as informações que permitem ao individuo processar sua concepção do suporte social estão organizadas em 3 classes de crenças: de que é amado e que existem pessoas preocupadas com ele; de que é apreciado e valorizado, e de que pertence a uma rede social.

Na visão de Rodriguez; Cohen (1998) existem diferentes tipos de apoio que alguém pode receber da rede social, sendo três os mais amplamente estudados: suporte emocional, suporte instrumental e suporte informacional (SIQUEIRA, 2008).

O Suporte emocional refere-se ao que as pessoas fazem e dizem a alguém (dar conselhos, ouvir seus problemas, mostrar-se empático e confiável) e é percebido como expressão de carinho, cuidados e preocupação do outro; o suporte instrumental compreende as ajudas tangíveis ou práticas que outros (pessoas ou instituições) podem prover a alguém (cuidados com crianças, provisões de transporte, empréstimos de dinheiro ou ajudas com tarefas diárias), e o suporte informacional inclui receber de outras pessoas noções indispensáveis para que o indivíduo possa guiar e orientar suas ações ao dar solução a um problema ou, no momento de tomar uma decisão (SIQUEIRA, 2008).

Reafirmando a importância do suporte social, Flynt; Wood; Scott (1992) discutem que muitas variáveis moderadoras podem atuar no processo de adaptação familiar ao estresse associado a ter uma criança com deficiência (MATSUKURA et al., 2007). Consideram que essas variáveis incluem o status da família antes do nascimento da criança, disponibilidade de recursos financeiros e suporte social, destacando que, dessas variáveis, o suporte social mostra ter o maior impacto no ajustamento familiar.

A relevância desse estudo para os fisioterapeutas, bem como para outros profissionais que trabalham com crianças com SD, é trazer dados concretos sobre a percepção do suporte social pelos cuidadores de crianças com SD, reconhecendo que atividades diárias são realizadas em um contexto social, com cuidadores que fornecem suporte e orientam o desempenho.

\section{Objetivo}

Conhecer a percepção do suporte social de cuidadores de crianças com SD em relação ao grau de satisfação e ao número de pessoas suportivas na região sul do estado de Minas Gerais, e estabelecer a relação entre os mesmos. 


\section{Método}

A população estudada foi composta por 50 adultos de ambos os gêneros, cuidadores de crianças com SD, com idade entre 6 meses a 7 anos e meio, frequentadores regulares de instituições especializadas.

Os cuidadores foram selecionados segundo os critérios de inclusão: ser alfabetizado, ser cuidador principal de uma criança com SD, estar vinculado a uma instituição de atendimento às pessoas com necessidades especiais, exercer a função de cuidador há, pelo menos, seis meses, estar disponível e consentir em participar da pesquisa e concordar em responder ao instrumento de coleta de dados, firmando o termo de consentimento. Foram critérios de exclusão: cuidadores de crianças com SD que tivessem outros diagnósticos associados, como autismo e encefalopatia crônica.

O estudo foi realizado em instituições de educação especial no Sul do Estado de Minas Gerais. Outras cidades foram contatadas, porém não houve possibilidade de coleta de dados pela indisponibilidade em receber a pesquisadora ou por não estarem matriculadas crianças na idade do estudo. A coleta de dados ocorreu em nove cidades cujo número de cuidadores coletados estão descritos na tabela 1.

Tabela 1: Cidades e o no de cuidadores coletados

\begin{tabular}{|l|c|}
\hline Cidades & Cuidadores \\
\hline Itajubá & 11 \\
\hline Caxambu & 5 \\
\hline Pouso Alegre & 10 \\
\hline Poços de Caldas & 8 \\
\hline Santa Rita do Sapucaí & 2 \\
\hline Três Corações & 6 \\
\hline Campanha & 2 \\
\hline Ouro Fino & 2 \\
\hline Lambari & 4 \\
\hline TOTAL & 50 \\
\hline
\end{tabular}

A coleta de dados foi realizada nas próprias instituições. Foi feito contato com a Diretora de cada instituição que promovia o encaminhamento para a Assistente Social, a qual realizava o levantamento das crianças com SD na faixa etária de 6 meses a 7 anos e meio. Posteriormente ao levantamento, obtinha-se das Diretoras o aval para a coleta na instituição. Novo contato com as Assistentes Sociais era feito para que fosse agendado dia e hora para a aplicação dos instrumentos de pesquisa na instituição, de acordo com a disponibilidade dos cuidadores.

Todos os participantes receberam a carta de informação ao sujeito e o termo de consentimento para assinatura. O mesmo se aplica às instituições, que assi- 
naram por meio de seus representantes, o termo de consentimento após a leitura da carta de informação. O projeto foi aprovado pelo Comitê de Ética em pesquisa sob o número CEP/UPM 1297/11/2010 e CAAE 0105.0.272.000-10.

Os instrumentos selecionados para este estudo foram o Social Support Questionnaire (SSQ), que fornece escores para o número de figuras de suporte percebido pelos respondentes e para a satisfação com o suporte social recebido. O SSQ é composto por 27 questões, sendo que cada questão solicita uma resposta em duas partes. Na primeira parte, deve ser indicado o número de fontes de suporte social percebido (SSQ-N), podendo o respondente listar até nove possibilidades (além da opção nenhum); na segunda parte, o respondente deve informar sobre sua satisfação com esse suporte (SSQ-S), fazendo uma opção em uma escala de 6 pontos (que varia de muito satisfeito a muito insatisfeito) (MATSUKURA; MARTURANO; OISHI, 2002). Foi utilizada, também, uma ficha de identificação para obtenção dos dados da criança e do cuidador (elaborada pelos pesquisadores).

A classificação da Associação Brasileira de Empresa de Pesquisa (ABEP) 2010 foi utilizada para determinar o nível sócio-econômico das famílias. Essa classificação da ABEP se baseia na acumulação de bens materiais, no poder aquisitivo e na escolaridade do chefe da família, agrupando os dados em cinco níveis socioeconômicos, que variam de A (mais elevado) a E (mais baixo). A aplicação é simples e os resultados são calculados a partir da soma da pontuação obtida. (Portal da ABEP).

\section{Resultados}

A amostra, composta por 50 cuidadores de crianças com SD, apresentou a maior concentração do nível sócio-econômico nas classes Cl, C2 e D (80\%), sendo apenas 20\% distribuído entre os níveis A2, B1, B2 e E (tabela 2). Ou seja, a grande maioria pertence a um nível sócio-econômico baixo.

Tabela 2: Caracterização do nível sócio-econômico dos cuidadores

\begin{tabular}{|c|c|c|}
\hline Classe & Cuidadores & (\%) \\
\hline $\mathrm{Al}$ & 0 & $0 \%$ \\
\hline $\mathrm{A} 2$ & 3 & $6 \%$ \\
\hline $\mathrm{B} 1$ & 2 & $4 \%$ \\
\hline $\mathrm{B} 2$ & 4 & $8 \%$ \\
\hline $\mathrm{Cl}$ & 13 & $26 \%$ \\
\hline $\mathrm{C} 2$ & 14 & $28 \%$ \\
\hline $\mathrm{D}$ & 13 & $26 \%$ \\
\hline $\mathrm{E}$ & 1 & $2 \%$ \\
\hline TOTAL & 50 & $100 \%$ \\
\hline
\end{tabular}


O cuidador principal foi em $92 \%$ dos casos a mãe, seguido dos avós (4\%), pai $(2 \%)$ e tia (2\%). A idade dos cuidadores variou entre 16 e 72 anos (média $=40$ anos) e o nível de escolaridade foi, em $46 \%$ dos casos, primário completo, seguido de ginasial completo $(28 \%)$, colegial completo $(18 \%)$ e superior completo $(8 \%)$. Entre os cuidadores, $20 \%$ exerciam atividade remunerada fora do ambiente doméstico. O estado civil foi, em 70\% dos casos, casados, seguido de união estável (18\%), solteiros $(8 \%)$ e separados ou divorciados (4\%).

O questionário que avalia o suporte social (SSQ) é composto por 27 questões dividas em duas partes: o SSQ-N, que considera o número de pessoas tidas como suportivas e o SSQ-S, que mede a satisfação com esse suporte.

Para o grupo estudado, a grande maioria das respostas indicativas de pessoas suportivas apontou a falta de uma pessoa de referência, sendo assinalada a resposta "ninguém". Em seguida, aparecem o marido, filho, pais, irmãos, amigos, esposa e médico da criança (Tabela 3).

Tabela 3: Frequência de respostas para o SSQ-N

\begin{tabular}{|c|c|c|}
\hline Pessoas Suportivas & Número de Respostas às 27 questões & Média simples \\
\hline Ninguém & 959 & 35,5 \\
\hline Marido & 178 & 6,6 \\
\hline Filho & 128 & 4,7 \\
\hline Pais & 98 & 3,6 \\
\hline Irmãos & 76 & 2,8 \\
\hline Amigos & 32 & 1,2 \\
\hline Esposa & 8 & 0,3 \\
\hline Médico & 4 & 0,1 \\
\hline TOTAL DE RESPOSTAS & 1483 & \\
\hline
\end{tabular}

O SSQ-S apresentou como resultado a média de 2,88 em uma escala entre zero a seis, na qual o zero representa muito insatisfeito e o seis representa muito satisfeito (Figura l). 
Figura 1 - Sumário estatístico para os escores do suporte social

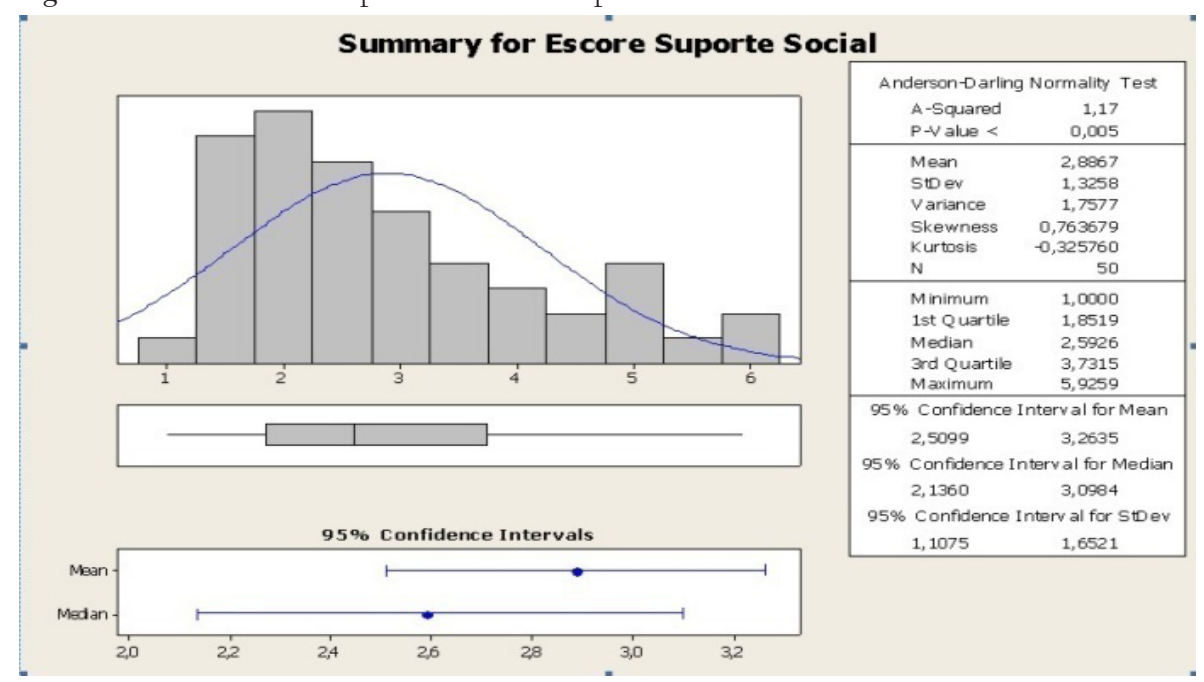

A princípio, a amostra foi analisada considerando duas faixas etárias distintas (crianças maiores de 4 anos e menores de 4 anos), pois suspeitava-se que o SSQ-N e SSQ-S pudessem ser diferentes em função da idade da criança. As amostras, em separado, não foram consideradas provenientes de uma população com distribuição normal (verificado através de teste de Anderson Darling). As medianas do SSQ-N e SSQ-S foram comparadas e os resultados para as duas faixas etárias puderam ser considerados como provenientes de população com mesma mediana (teste de Mann - Whitney com nível de significância de 5\%). A partir desse ponto, os resultados dos questionários foram analisados como uma amostra única.

Para analisar a relação entre o número de pessoas suportivas e o suporte social percebido, primeiramente, foi feita uma análise para verificar se todo o grupo de cuidadores poderia ser considerado homogêneo, desconsiderando-se a faixa etária, comparando-se as amostras de cuidadores de crianças com idade acima de 4 anos e abaixo de 4 anos. Através do teste de Anderson-Darling, ao nível de significância de $5 \%$, rejeita-se a hipótese da normalidade das amostras. Portanto, foram utilizados testes não paramétricos. O teste de Mann-Whitney comparou as medianas do número pessoas suportivas ao nível de significâncias de 5\% e as mesmas não podem ser consideradas diferentes para os dois grupos $(\mathrm{p}$-value $=0,236)$.

O mesmo acontece na comparação das medianas do grau de satisfação do suporte social, ao nível de significância de 5\% as medianas não são consideradas diferentes para os dois grupos $(\mathrm{p}$-value $=0,105)$. 
Assim, os cuidadores constituirão uma única amostra na análise da relação entre número de pessoas suportivas x suporte social percebido. O sumário estatístico dessa amostra é apresentado para cada variável na figura 2.

Figura 2 - Sumário estatístico para o número de pessoas suportivas

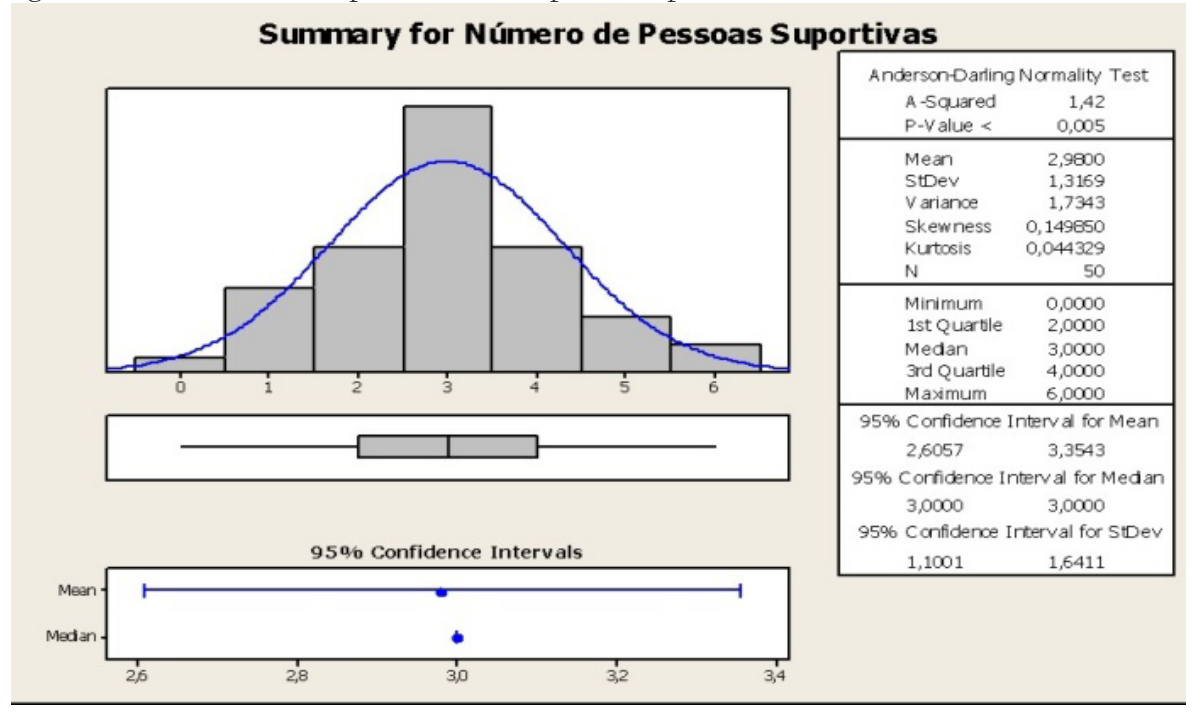

O diagrama de dispersão apresentado ,na Figura 3, mostra a relação entre o suporte social e o número de pessoas suportivas. O índice de correlação de Spearman apresenta um valor de 0,4l, o que indica uma correlação entre fraca e moderada, estatisticamente significativa ao nível de 0,05 (valor $\mathrm{P}=0,003$ ).

Figura 3 - Diagrama de dispersão mostrando a relação entre o suporte social e o número de pessoas suportivas.

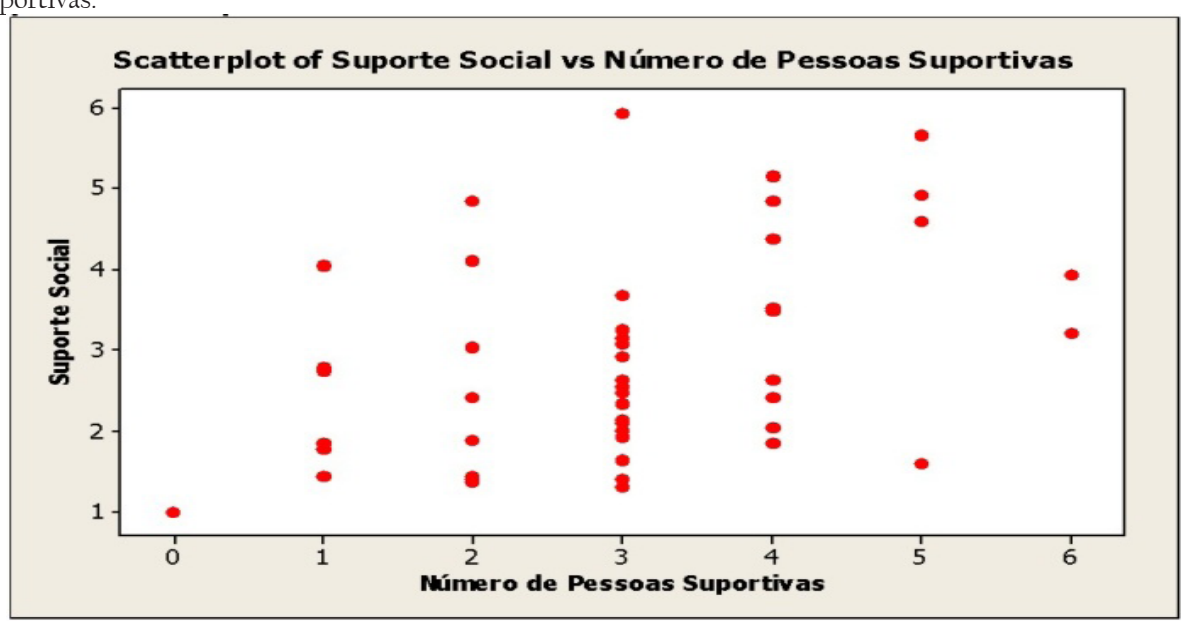




\section{Discussão}

É possível supor que, no contexto brasileiro, a dificuldade de sobrevivência, a educação deficiente e a baixa renda per capita são fatores que desfavorecem a mobilização da população frente ao suporte social de cuidadores de crianças com SD. O presente estudo mostrou que os cuidadores, em sua maioria, têm um nível sócio - econômico e de escolaridade baixos e, a maioria deles, não exerce atividade remunerada fora do ambiente doméstico. Essa situação poderia ser modificada por meio da implementação de políticas públicas que promovam assistência, apoio e proteção social a esses cuidadores.

No contexto familiar, a pessoa que assume o papel de cuidador está sujeita à produção de demandas de cuidados que afetam sua dimensão física, mental e social. Em estudo de Pazin \& Martins (2007), os cuidadores assumiram totalmente a carga de cuidados das crianças e eram, na sua maioria, mulheres, casadas, conforme também observado em nosso estudo. Os cuidadores, preferencialmente, são as mães que vivem junto à criança e que têm proximidade afetiva, dados também observados em outros estudos e ratificados com nosso estudo.

A sobrecarga imposta à mãe, uma vez que se trata de um filho com SD, indiscutivelmente requer mais tempo, atenção e cuidados do que uma criança não especial. Estudos sugerem que a divisão das tarefas com o pai e os demais familiares proporcionará melhor relacionamento entre eles, minimizando, assim, os sentimentos de ciúmes e competição. Estudo de Pazin \& Martins (2007), constatou que as dimensões mais comprometidas na qualidade de vida desses cuidadores foram o isolamento, o que também observamos em nosso estudo que apontou a falta de uma pessoa de referência, sendo assinalada como pessoa suportiva a resposta "ninguém". Em seguida, aparecem o marido, filho, pais, irmãos, amigos, esposa e médico da criança.

Estudos evidenciaram que os cuidadores dessas crianças, ao assumir sozinho os cuidados, manifestam, freqüentemente, seu desconforto e sentimento de solidão, quando não sentem apoio de outros membros da família. A necessidade de dividir com outras pessoas o desgaste provocado pelas situações de enfrentamento de eventos negativos indica a vontade de suavizar o impacto provocado pela carga de tarefas.

É importante que o cuidador possa receber apoio de pessoas da família, pois a exposição prolongada a uma situação potencialmente geradora de estresse contribui fortemente para o esgotamento geral do indivíduo e seu conseqüente sentimento de sobrecarga pelos efeitos psicossociais da síndrome.

Estudo apresentado por Deslandes \& Bastos (2008), evidenciou uma maior vulnerabilidade, que pode levar as famílias a grandes dificuldades para a reabilitação de seus filhos. Ainda, segundo Deslandes \& Bastos (2008), vários autores 
analisam que, nas últimas décadas, tem-se explorado a importância do apoio social na recuperação de saúde, que facilita o enfrentamento dos problemas e a diminuição dos efeitos negativos de situações estressantes, contribuindo para o bem-estar geral.

Nesse estudo de Deslandes \& $\&$ Bastos (2008), observou-se que o apoio emocional e o suporte financeiro, obtidos através das práticas relacionadas à fé ou pela rede composta por amigos, parentes ou na vizinhança, constituem lógicas de atenção que se distinguem do mercado ou da regulação do Estado. Constitui-se uma rede de ajuda mútua, capaz de fornecer alternativas para que se enfrentem as condições de pobreza, sendo capaz de romper algumas das barreiras de exclusão social. No estudo de Barbosa et al (2009), foi possível identificar que a família ampliada e os amigos são uma importante fonte de suporte emocional, ajudando a família a se sentir mais confortada e amparada. Igualmente, em nosso estudo, notamos como integrantes da rede de apoio social, pessoas da família e com exceção um profissional da saúde (médico da criança) como integrante da rede de apoio social.

O estudo de Rezende, Mendes \& Santos (2007) causou surpresa pela ausência de citação pelos idosos dessas equipes como agente de suporte social, além do desconhecimento do nome da maioria dos profissionais de saúde que lhes prestavam assistência no âmbito hospitalar. Este fato vem mostrar a necessidade de uma análise crítica por parte dos profissionais, cujas ações exigem novas posturas como o compartilhamento, e a consideração com a comunidade com a qual trabalham.

A análise acerca do grau de satisfação em relação aos apoios recebidos (que variava de muito insatisfeito, insatisfeito, nem satisfeito nem insatisfeito, satisfeito e muito satisfeito), no estudo de Rezende, Mendes \& Santos (2007), demonstrou que a maioria das respostas ficou entre os itens quatro e cinco, apontando que os idosos estavam satisfeitos e muito satisfeitos com o apoio recebido, a exemplo de outros estudos. Aquelas mães que puderam contar com uma rede social de suporte reconheceram a sua importância como colaboradora no cuidado dos filhos com deficiência. A participação de vizinhos, companheiros de igreja e familiares também não deixa de atestar a ordenação da rotina e da identidade desta cuidadora, agora foco da atenção e apoio coletivos

Nesse estudo foi possível perceber que os cuidadores, em sua maioria mães (92\%), mostram satisfação entre fraca e moderada denotando que essas cuidadoras não puderam contar com uma rede de suporte social eficiente no cuidado dos filhos com SD, ou seja, percebem pouco apoio social. Excepcionalmente, foi destacada a participação do marido para além do papel de prover o sustento, e de outros filhos e demais familiares para que ajudassem nos cuidados cotidianos, aliviando a sobrecarga do trabalho. Esta é a realidade para a maior parte das mães que pertencem às camadas populares. Isto vai de encontro à observação de Waldman et. al. (1999), que detectaram que os pais são muito menos envolvidos que as mães nos cuidados com os filhos, especialmente quando eles têm deficiência. 
Em relação ao grupo estudado, observou-se o contrário, que os maridos foram tidos como a referência mais importante de apoio em seu cuidado com as crianças com SD, seguido dos filhos, pais, irmãos, amigos, esposa e médico da criança. Esse dado vem corroborar com o estado civil da maioria dos cuidadores que são casados (70\%), seguido de união estável (18\%), o que nos faz pensar que os pais estão mais atuantes e presentes no cuidado e assistência com o filho com SD.

\section{Conclusão}

Esse estudo ressalta que as famílias das crianças com SD precisam ser incluídas no planejamento do cuidado e no processo de reabilitação da criança, e também requerem cuidados. Os cuidadores do estudo, seus filhos e suas famílias necessitam da atenção, do respeito, do acolhimento, de informações e das orientações da equipe de saúde. Os profissionais da saúde precisam estar atentos às necessidades das famílias e se dispor a ouvir e a compreender seus temores, suas dificuldades e inquietações, buscando formas de facilitar a adaptação e a convivência com o filho com $\mathrm{SD}$, como parte de seu trabalho com elas.

É importante que os pais possam buscar informações nas instituições especializadas, organizando-se em grupos, para encontrar os melhores caminhos e informações atualizadas sobre a SD, além do apoio e orientações necessárias para o pleno desenvolvimento do seu filho. A instituição proporciona, também, o encontro entre mães e pais que se apóiam mutuamente, auxiliando na superação dos conflitos que surgem inicialmente e ao longo do desenvolvimento da criança. Para tanto, é necessário que as famílias sejam orientadas e encaminhadas pelos profissionais que realizam os primeiros contatos, buscando-se, assim, um trabalho interdisciplinar que melhor atenda as necessidades dos familiares e facilite o desenvolvimento da criança e o trabalho de toda a equipe. Torna-se claro que iniciativas de trabalhos que considerem as necessidades familiares de suporte social podem trazer contribuição à área da educação especial, uma vez que o contexto da mesma compreende o atendimento educacional especializado com participação da família em articulação com as demais políticas públicas (GARCIA; MICHELS, 2011).

Consideramos pertinente o incremento de ações e incentivos governamentais e não-governamentais para o desenvolvimento de equipes profissionais e de instituições especializadas, voltadas ao atendimento, à reabilitação e a inclusão de crianças com SD e suas famílias na sociedade. As famílias precisam de programas de apoio apropriados para potencializarem ao máximo suas próprias capacidades. Seus direitos e o dos seus filhos configuram-se na igualdade de acesso contínuo ao espaço comum da vida em sociedade e a todo tipo de oportunidade. Intervenções que contextualizem a vida delas, na comunidade, são essenciais, no sentido de promover as adequações para que possam adquirir condições de inclusão e de vida na sociedade.

Conclui-se que os cuidadores dessas crianças, e seu contexto ecológico devam ser melhores estudados, pois a compreensão e o conhecimento do impacto na qualidade de vida dessas pessoas e a conseqüência desse na assistência às crianças com SD, por eles cuidadas, podem estabelecer, para a equipe interdisciplinar, planejamentos terapêuticos mais eficazes. 


\section{Referências}

ALDERSON, P. Down's syndrome: cost, quality and value of life.5.ed. Soc Sci Med, 2001. 627-38 p. ASSOCIAÇÃO BRASILEIRA DE EMPRESAS DE PESQUISA. Portal da ABEP. Site www.abep.org.br

BARBOSA, M.A.M. et al. Cuidado da criança com deficiência: suporte social acessado pelas mães. Porto Alegre, Rev. Gaúcha Enferm, 2009.

BASTOS, O.M. ; DESLANDES, S.F. A experiência de ter um filho com deficiência mental: narrativas de mães. 9. ed. Cad. Saúde Pública, Rio de Janeiro, 2008.

BRASIL. Decreto nº 6949/ 2009. Promulga a Convenção Internacional sobre os Direitos das Pessoas com Deficiência e seu Protocolo Facultativo assinados em Nova York em 30 de março de 2007.

BRASIL. Ministério da Educação. Lei de Diretrizes e Bases da Educação (Lei 9.394/96). Brasília, 1996.

Ministério da Educação. Política Nacional de Educação Especial na perspectiva da educação inclusiva. Ministério da Educação. Brasília, 2008. Acesso em: 18 mar. 2013. 15p.

BUZATTO, L. L.; BERESIN, R. Qualidade de vida dos pais de crianças portadoras da SD. 2.ed. Einstein: São Paulo, 2008.

COBB, S. Social support as a moderator of life stress. 5.ed. Psychosomatic Medicine, 1976. p. 300-14.

CORRICE, A.M.; GLIDDEN, L.M. The Down syndrome advantage: fact or fiction? Am J Intellect Dev Disabil, 4.ed. 2009.

COUTO, T. H. A. M.; TACHIBANA, M.; AIELLO-VAISBERG, M. J. A mãe, o filho e a SD. 37.ed. Paidéia, Ribeirão Preto, 2007.

CUNHA, A. et al. Impacto da notícia da síndrome de Down para os pais: histórias de vida. Rio de Janeiro, Ciênc.saúde coletiva, 2010.

DESSEN, M. A.; SILVA, N. L. P. Deficiência mental e família: uma análise da produção científica. Paidéia: Ribeirão Preto, 2000.

FLYNT, S. W.; WOOD, T. A.; SCOTT, R. L. Social of mothers of children with mental retardion. 4.ed. Mental Retardation, 1992.

GARCIA, R.M.C.; MICHELS, M.H. A política de educação especial no Brasil (1991-2011): uma análise da produção do GT15 - educação especial da ANPED. Rev. bras. educ. espec. [online], vol. 17, 2011.

MATSUKURA, T. S. et al. Estresse e suporte social em mães de crianças com necessidades especiais. Revista Brasileira de Educação Especial, 2007.

; MARTURANO, E. M.; OISHI, J. O Questionário de Suporte Social (SSQ): estudos de adaptação para o português. 5.ed. Revista Latino Americana de Enfermagem, Ribeirão Preto, 2002.

MAZZOTTA, M.J.S. Trabalho docente e formação de professores de educação especial. São Paulo: EPU, 1993.

MOST, D. E. et al. Stress trajectories in mothers of young children with Down syndrome. J Intellect Disabil Res, 2006.

PAN, M. O direito à diferença: uma reflexão sobre deficiência intelectual e educação inclusiva. Curitiba: IBPEX, 2008. 
PAZIN, A. C.; MARTINS, M.R.I. Desempenho funcional de crianças com Síndrome de Down e a qualidade de vida de seus cuidadores . 4.ed. Rev.Neurocienc, 2007.

PEREIRA-SILVA, N .L., DESSEN, M. A. Famílias de crianças com SD: sentimentos, modos de vida e estresse parental. 2.ed. Interação em Psicologia, 2006.

REZENDE, L. K.; MENDES, I. J. M.; SANTOS, B. M. O. Suporte social para idosos portadores de insuficiência cardíaca. 1.ed. Rev. Ciênc. Farm. Básica Apl, 2007.

RODRIGUEZ, M. S.; COHEN, S. Social support. 3.ed. Encyclopedia of Mental Health, 1998.

SIQUEIRA, M. M. M. Construção e validação da Escala de Percepção de Suporte Social. 2.ed. Psicologia em Estudo, Maringá, 2008.

SLUZKI C. E. A rede social na prática sistêmica: alternativas terapêuticas. São Paulo: Casa do Psicólogo, 1997.

SUNELAITIS, R. C.; ARRUDA, D.C.; MARCON , S. S. A repercussão de um diagnóstico de SD no cotidiano familiar: perspectiva da mãe. 3. ed. Acta Paul Enferm, 2007.

WALDMAN, H.B.; SWERDLOFF, M.; PERLMAN, S.P. Children with mental retardation grow older. 4.ed. ASDC J Dent Child, 1999.

WEHMEYER, M. L. et al. Perspectives: The intellectual disability construct and its relation to human functioning. Intelectual and Developmental Disabilities. Volume 46, Number 4: 311-318/ August 2008.

WISEMAN, F. K.; ALFORD, K. A.; TYBULEWICZ, V. L. Down syndrome-recent progress and future prospects. 1.ed. Hum Mol Genet, 2009.

\section{Nota}

${ }^{1}$ Este artigo foi desenvolvido em cidades do interior do Estado de Minas Gerais, em Instituições de atendimento especializados a crianças com síndrome de Down e financiado em parte pelo Fundo Mackenzie de Pesquisa. Nada a declarar sobre conflito de interesses.

\section{Correspondência}

Luciana Krauss Rezende - Av. Dr.Antônio Braga Filho, 687, Bairro: Varginha, CEP 37500-032, Itajubá, Minas Gerais, Brasil.

E-mail: lukrare@uol.com.br-silvanablascovi@mackenzie.br - barca@unifei.edu.br

Recebido em 23 de julho de 2012

Aprovado em 04 de abril de 2013 
